\title{
ON THE UNICITY CONJECTURE FOR GENERALIZED MARKOFF EQUATION
}

\author{
Youb Raj Gaire \\ Email Address: yrgaire@yahoo.com \\ Central Department of Mathematics, T.U., Nepal
}

\begin{abstract}
The Markoff equation $x^{2}+y^{2}+z^{2}=3 x y z$ is introduced by A.A. Markoff in 1879. A famous conjecture on the Markoff equation, made by Frobinus in 1913, states that any Markoff triples (x, y, z) with $\mathrm{x} \leq \mathrm{y} \leq \mathrm{z}$ is uniquely determined by its largest number $z$. The complete solution of this equation is still open however the partial solution is given by Barager (1996), Button (2001), Zhang (2007), Srinivasan (2009), Chen and Chen (2013).

In 1957, Mordell developed a generalization to the Markoff equation of the form $x^{2}+y^{2}+z^{2}=A x y z+B$ where, $A$ and $B$ are positive integers. In 2015, Donald McGinn take a particular form of above equation with $\mathrm{A}=1$ and $\mathrm{B}=\mathrm{A}$ and gave a partial solution to the unicity conjecture to this equation. In this paper, the partial solution to the unicity conjecture to the equation of the form $\mathrm{x}^{2}+\mathrm{y}^{2}+\mathrm{z}^{2}=3 \mathrm{xyz}+\mathrm{A}$ where $\mathrm{A}$ is positive integer with $\mathrm{A} \leq 4\left(\mathrm{x}^{2}-1\right)$ is given.
\end{abstract}

\section{Introduction}

The Markoff equation

$$
\mathrm{x}^{2}+\mathrm{y}^{2}+\mathrm{z}^{2}=3 \mathrm{xyz}
$$

was established in 1879 by A.A. Markoff (see [C1]). A triples (x, y, z) where $\mathrm{x}, \mathrm{y}, \mathrm{z}$ are positive integers and $\mathrm{x} \leq \mathrm{y} \leq \mathrm{z}$; is called Markoff triple if it satisfies the above Markoff equation and the numbers $\mathrm{x}, \mathrm{y}$ and $\mathrm{z}$ are called Markoff numbers.. If $(\mathrm{x}, \mathrm{y}, \mathrm{z})$ is a Markoff triple then we easily drawn others with the help of the transformation

$$
\begin{aligned}
& \phi:(\mathrm{x}, \mathrm{y}, \mathrm{z}) \rightarrow(\mathrm{x}, \mathrm{z}, 3 \mathrm{xz}-\mathrm{y}), \\
& \psi:(\mathrm{x}, \mathrm{y}, \mathrm{z}) \rightarrow(\mathrm{y}, \mathrm{z}, 3 \mathrm{yz}-\mathrm{x}) .
\end{aligned}
$$

In this way, we can obtain infinitely many solution of Markoff equation, which we also known as Markoff triples. In ascending order the first 10 Markoff triples are $(1,1,1),(1,1,2),(1,2,5),(1,5$, 13), $(2,5,29),(1,13,34),(1,34,89),(2,29,169),(5,13,194),(1,89,233)$ and the first 10 Markoff numbers are $1,2,5,13,29,34,89,169,194,233$. It is easy to see that if $(\mathrm{x}, \mathrm{y}, \mathrm{z})$ is a Markoff triple with

$\mathrm{x} \leq \mathrm{y} \leq \mathrm{z}$ and $\mathrm{z}>2$ then $\mathrm{x}<\mathrm{y}<\mathrm{z}$. Clearly the number $\mathrm{z}$ is the largest Markoff number in the triple. In 1913, Frobenius [see Z1] made a conjecture on the unicity of such Markoff number z, which is still open. He conjectured the problem as:

Unicity Conjecture of Markoff Equation [Z1]: If $\left(\mathrm{x}_{1}, \mathrm{y}_{1}, \mathrm{z}\right)$ and $\left(\mathrm{x}_{2}, \mathrm{y}_{2}, \mathrm{z}\right)$ are two Markoff triples where $\mathrm{x}_{1}<\mathrm{y}_{1}<\mathrm{z}$ and $\mathrm{x}_{2}<\mathrm{y}_{2}<\mathrm{z}$ then $\mathrm{x}_{1}=\mathrm{x}_{2}$ and $\mathrm{y}_{1}=\mathrm{y}_{2}$.

The first partial solution to this conjecture was given by Barager [B1] in 1996. He proved that if $\mathrm{z}<10^{140}$ or if either $\mathrm{z}, 3 \mathrm{z}-2$ or $3 \mathrm{z}+2$ is a prime, twice a prime or four times a prime then $\mathrm{z}$ is unique. In 1996, Schmutz [C1] proved that if $\mathrm{z}$ is a prime power then $\mathrm{z}$ is unique. In 2001, by using some techniques on algebraic number field, Button [B2] showed that any $\mathrm{z}$ of the form $k p^{l}$ with $\mathrm{k} \leq 10^{35}$ and $\mathrm{p}$ is a prime must be unique. In 2007, Zhang [Z1] proved that a Markoff number $\mathrm{z}$ is unique if one of $(3 z-2)$ or $(3 z+2)$ is of the form $k p^{l}$ where $\mathrm{k}$ is either 1 or 4 or 8 and $p^{l}$ is a prime power. In 2009, A. 
Srinivasan [S1] also gave a partial solution to the unicity conjecture of Markoff equation. In 2013, Chen and Chen [C1] proved that if either $(3 z-2)$ or $(3 z+2)$ is of the form $\mathrm{kp}^{\ell}$, where $k$ is an integer with $1 \leq \mathrm{k} \leq 10^{10}$ and $\mathrm{p}^{\ell}$ is a prime power, then $\mathrm{z}$ unique.

In 1957, Mordell gave a generalization of Markoff equation in the form $x^{2}+y^{2}+z^{2}=A x y z+B$ where $\mathrm{A}$ and $\mathrm{B}$ are integer values. This equation is similar to the generalized Markoff equation of the form $x^{2}+y^{2}+z^{2}=x y z+A$ due to D. Maginn [D1]. He gave a partial solution to the generalized Markoff equation by using Chebyshev polynomial [D1], which was published in 2015. If we choose $A=3$ for the Mordell's generalization then gives $x^{2}+y^{2}+z^{2}=3 x y z+A$ as a particular case of Mordell's generalization with $\mathrm{B}=\mathrm{A}$. In this paper, the partial solution of the unicity conjecture to the equation of the form $x^{2}+y^{2}+z^{2}=3 x y z+A$ where $A$ is positive integer value satisfies the condition $A \leq 4\left(x^{2}-\right.$ $1)$.

\section{Some Results on Unicity Conjecture:}

This section includes some lemmas.

Lemma -1 [C1]: Let A, B and $C$ are integers with $B>0$. Suppose that $d$ is a positive integer with $\mathrm{d} \mid\left(\mathrm{A}^{2} \mathrm{~B}-\mathrm{C}^{2}\right)$. If $(+\mathrm{C})$ is non zero and $\mathrm{d} \leq 2|\mathrm{~A}| \sqrt{\mathrm{B}}+1$ then

$$
|\mathrm{A} \sqrt{\mathrm{B}}+\mathrm{C}| \geq \frac{\mathrm{d}}{\mathrm{A} \sqrt{\mathrm{B}}+1} .
$$

Lemma -2 [C1]: Let A, B, C, $M$ and $N$ be integers with $M>0$ and $N>0$. Suppose that $d$ is a positive integer with $d \mid \quad\left(\left(A^{2} M+B^{2} N-C^{2}\right)^{2}-4 A^{2} B^{2} M N\right)$ and $d \leq 4|A B| \sqrt{M N}+1$. If $\mathrm{A} \sqrt{\mathrm{M}}+\mathrm{B} \sqrt{\mathrm{N}}+\mathrm{C} \neq 0$ then

$$
|A \sqrt{M}+B \sqrt{N}+C| \geq \frac{d}{(2|A| \sqrt{M}+2|B| \sqrt{N}+1)(4|A B| \sqrt{M N}+1)} .
$$

Lemma -3 [C1]: Let $A, B, C, M$ and $N$ be integers with $M>0$ and $N>0$ such that neither $M$ nor $N$ is a square. Then $A \sqrt{M}+B \sqrt{N}+C=0$ if and only if $A \sqrt{M}+B \sqrt{N}=0$ and $C=0$.

Lemma -4: If $z>2$ and if $A \leq 4\left(x^{2}-1\right)$ then

(i) $\quad \frac{\mathrm{z}}{3 \mathrm{x}}-\frac{\mathrm{A}}{3 \mathrm{xz}}<\mathrm{y}<\frac{\mathrm{z}}{3 \mathrm{x}-1} ; \mathrm{x} \leq \sqrt{\frac{\mathrm{z}}{5}}$

$$
y=\frac{1}{2}\left(3 x-\sqrt{9 x^{2}-4-A}\right) z+r ; \frac{x}{3 z}<r<\frac{x}{2 z} .
$$

Proof: By

$$
\begin{aligned}
& 3 x y z+A=x^{2}+y^{2}+z^{2}>z^{2} \\
& \Rightarrow y>\frac{z}{3 x}-\frac{A}{3 x z}
\end{aligned}
$$

Let $\mathrm{z}^{\prime}=3 \mathrm{xy}-\mathrm{z}$ then for $\mathrm{z}>2$ and $\mathrm{x}<\mathrm{y}<\mathrm{z}$,

$$
\begin{aligned}
(z-y)\left(z^{\prime}-y\right) & =z z^{\prime}-\left(z+z^{\prime}\right) y+y^{2} \\
& =z(3 x y-z)-3 x y^{2}+y^{2} \\
& =x^{2}+y^{2}-A-3 x y^{2}+y^{2} \\
& <3 y^{2}-2 x y^{2}-A
\end{aligned}
$$




$$
\begin{aligned}
& =3 y^{2}(1-x)-A \\
& \leq 0 ;(\text { for } x \geq 1)
\end{aligned}
$$

Since $\mathrm{y}<\mathrm{z}$, so

$$
\begin{aligned}
z^{\prime}<y & \Rightarrow 3 x y-z<y \\
& \Rightarrow y<\frac{z}{3 x-1}
\end{aligned}
$$

Thus from (1) and (2),

$$
\frac{\mathrm{z}}{3 \mathrm{x}}-\frac{\mathrm{A}}{3 \mathrm{xz}}<\mathrm{y}<\frac{\mathrm{z}}{3 \mathrm{x}-1}
$$

Since $A>0$. So, the equation $\mathrm{x}^{2}+\mathrm{y}^{2}+\mathrm{z}^{2}=3 \mathrm{xyz}+\mathrm{A}$ has no integer solution for $1 \leq \mathrm{x}<\mathrm{y}<\mathrm{z}<5$. So, suppose $z \geq 5$. Then the second inequality of (i) is true for $x=1$. Now suppose $x \geq 2$. If $x<z^{\prime}$ then ( $x$, $z^{\prime}, y$ ) is a Markoff triple with $x<z^{\prime}<y$. Then by the first inequality of (3) we have,

$$
\mathrm{x}<\mathrm{z}^{\prime}<\frac{\mathrm{y}}{3 \mathrm{x}-1}<\frac{\mathrm{z}}{(3 \mathrm{x}-1)^{2}}
$$

If $\mathrm{z}^{\prime} \leq \mathrm{x}$ then $\left(\mathrm{z}^{\prime}, \mathrm{x}, \mathrm{y}\right)$ is a Markoff triple with $\mathrm{z}^{\prime} \leq \mathrm{x}<\mathrm{y}$. For $2 \leq \mathrm{x}<\mathrm{y}$ and by (3) we have,

$$
\mathrm{x}<\frac{\mathrm{y}}{3 \mathrm{z}^{\prime}-1}
$$

Then by $[\mathrm{C} 1]$ the inequality $\mathrm{x} \leq \sqrt{\frac{\mathrm{z}}{5}}$ holds. And, the equation $\mathrm{x}^{2}+\mathrm{y}^{2}+\mathrm{z}^{2}=3 \mathrm{xyz}+\mathrm{A}$ has a solution,

$$
y=\frac{1}{2}\left(3 x z-\sqrt{9 x^{2} z^{2}-4 z^{2}-4 x^{2}-A}\right)
$$

being $\mathrm{y}<\mathrm{z}$.

Let,

$$
y=\frac{1}{2}\left(3 x-\sqrt{9 x^{2}-4-A}\right) z+r
$$

where

$$
\begin{aligned}
r & =\frac{1}{2}\left(z \sqrt{9 x^{2}-4-A}-\sqrt{9 x^{2} z^{2}-4 z^{2}-4 x^{2}-A}\right) \\
& =\frac{2 x^{2}}{z \sqrt{9 x^{2}-4-A}+\sqrt{9 x^{2} z^{2}-4 z^{2}-4 x^{2}-A}}
\end{aligned}
$$

Since,

and

$$
\sqrt{9 \mathrm{x}^{2} \mathrm{z}^{2}-4 \mathrm{z}^{2}-4 \mathrm{x}^{2}-\mathrm{A}}<3 \mathrm{xz}
$$

Then (6) follows

$$
z \sqrt{9 x^{2}-4-A}<3 x z
$$

$$
r>\frac{2 x^{2}}{6 x z} \Rightarrow r>\frac{x}{3 z}
$$

Also, for $\mathrm{x} \geq 1$, and for $\mathrm{A} \leq 4\left(\mathrm{x}^{2}-1\right)$,

$$
\sqrt{9 x^{2} z^{2}-4 z^{2}-4 x^{2}-A} \geq \sqrt{9 x^{2} z^{2}-4-A}>\sqrt{5 x^{2} z^{2}}>2 x z
$$


and

$$
z \sqrt{9 x^{2}-4-A} \geq z \sqrt{5 x^{2}}>2 x z
$$

Then (6) implies,

$$
\mathrm{r} \leq \frac{\mathrm{x}}{2 \mathrm{z}}
$$

This completes the proof of lemma -4 .

Lemma -5: If $\left(\mathrm{x}_{\mathrm{i}}, \mathrm{y}_{\mathrm{i}}, \mathrm{z}\right)$ (for $\left.\mathrm{i}=1,2\right)$ are two Markoff triples with $\mathrm{x}_{\mathrm{i}}<\mathrm{y}_{\mathrm{i}}<\mathrm{z}$ and $\mathrm{x}_{1}<\mathrm{x}_{2}$ then
(i) $\mathrm{y}_{1}+\mathrm{x}_{1}>\mathrm{y}_{2}+\mathrm{x}_{2}$,
(ii) $\mathrm{y}_{1}>\mathrm{y}_{2}$,
(iii) $\mathrm{y}_{1}-\mathrm{x}_{1}>\mathrm{y}_{2}-\mathrm{x}_{2}$

Proof: By (4) we have,

$$
y=\frac{1}{2}\left(3 x z-\sqrt{9 x^{2} z^{2}-4 z^{2}-4 x^{2}-A}\right)
$$

and by lemma -4 , we have $x \leq \sqrt{\frac{z}{5}}$. So, we may define a function

$$
f(t)=3 z t-\sqrt{9 t^{2} z^{2}-4 z^{2}-4 t^{2}-A}+2 t .
$$

Then

$$
f^{\prime}(t)=3 z+2-\frac{9 t z^{2}-4 t}{\sqrt{9 t^{2} z^{2}-4 t^{2}-4 z^{2}-A}} .
$$

Clearly $\mathrm{f}^{\prime}(\mathrm{t})<0$ for $1 \leq \mathrm{t} \leq \sqrt{\frac{\mathrm{z}}{3}}$. This means $\mathrm{f}(\mathrm{t})$ is monotonically decreasing for $1 \leq \mathrm{t} \leq \sqrt{\frac{\mathrm{z}}{3}}$.

Therefore,

$$
\begin{array}{ll}
f\left(x_{1}\right)>f\left(x_{2}\right) & \text { for } x_{1}<x_{2} . \\
\text { i.e. } y_{1}+x_{1}>y_{2}+x_{2} &
\end{array}
$$

Since $\mathrm{x}_{1}<\mathrm{x}_{2}$, so

$$
\mathrm{y}_{1}>\mathrm{y}_{2} \text { and } \mathrm{y}_{1}-\mathrm{x}_{1}>\mathrm{y}_{2}-\mathrm{x}_{2} .
$$

This completes the proof of lemma -5 .

\section{The Unicity Theorem}

In this section we gave theorems on the unicity conjecture for generalized Markoff equation $\mathrm{x}^{2}+\mathrm{y}^{2}+\mathrm{z}^{2}=3 \mathrm{xyz}+\mathrm{A}$ for A is positive integer. The following theorems deal only the partial solution of the conjecture.

Theorem -6: Let $\varepsilon, \delta \in-1,1$. If $\left(\mathrm{x}_{1}, \mathrm{y}_{1}, \mathrm{z}\right)$ and $\left(\mathrm{x}_{2}, \mathrm{y}_{2}, \mathrm{z}\right)$ are two distinct Markoff triples such that $\mathrm{x}_{\mathrm{i}}<\mathrm{y}_{\mathrm{i}}<\mathrm{z}($ for $\mathrm{i}=1,2)$ then

$$
(2 \mathrm{z}+2 \varepsilon)^{1 / 14}<\operatorname{gcd}\left(\mathrm{y}_{1}+\varepsilon \mathrm{x}_{1}+\varepsilon(\mathrm{y} 2+\varepsilon \mathrm{x} 2) ; 3 \mathrm{z}+2 \varepsilon\right)<(3 \mathrm{z}+2 \varepsilon)^{13 / 14} .
$$

Proof: If possible suppose that $\left(\mathrm{x}_{1}, \mathrm{y}_{1}, \mathrm{z}\right)$ and $\left(\mathrm{x}_{2}, \mathrm{y}_{2}, \mathrm{z}\right)$ are two distinct Markoff triples with $\mathrm{x}_{1}=\mathrm{x}_{2}$. Then by the equation $\mathrm{x}_{\mathrm{i}}^{2}+\mathrm{y}_{\mathrm{i}}^{2}+\mathrm{z}^{2}=3 \mathrm{x}_{\mathrm{i}} \mathrm{y}_{\mathrm{i}} \mathrm{z}+\mathrm{A}($ for $\mathrm{i}=1,2)$, we have

$$
\begin{aligned}
\mathrm{y}_{1}{ }^{2}-\mathrm{y}_{2}{ }^{2} & =3 \mathrm{x}_{1} \mathrm{z}\left(\mathrm{y}_{1}-\mathrm{y}_{2}\right) \\
\Rightarrow \mathrm{y}_{1}+\mathrm{y}_{2} & =3 \mathrm{x}_{1} \mathrm{z}
\end{aligned}
$$


But we have $\mathrm{x}<\mathrm{y}<\mathrm{z}$, it follows that

$$
\mathrm{y}_{1}+\mathrm{y}_{2}<2 \mathrm{z}<3 \mathrm{z} \leq 3 \mathrm{x}_{1} \mathrm{z} .
$$

This leads a contradiction. So we should have $\mathrm{x}_{1} \neq \mathrm{x}_{2}$.

For simplicity, we fix $\varepsilon=\delta=1$ and $\mathrm{x}_{1}<\mathrm{x}_{2}$.

Let,

$$
\begin{aligned}
& \operatorname{gcd}\left(\mathrm{x}_{1}+\mathrm{x}_{2}+\mathrm{y}_{1}+\mathrm{y}_{2}, 3 \mathrm{z}+2\right)=\mathrm{m}, \\
& \mathrm{x}_{1}+\mathrm{x}_{2}+\mathrm{y}_{1}+\mathrm{y}_{2}=\mathrm{Tm}, \\
& \text { and } \quad 3 \mathrm{z}+2=\mathrm{mn} .
\end{aligned}
$$

For $\mathrm{z} \geq 10^{140}$, we observe that $\mathrm{mn}>10^{140}$.

By lemma -4 , we have

$$
y_{i}=\frac{1}{2}\left(3 x_{i}-\sqrt{9 x_{i}^{2}-4-A}\right) z+r_{i}, \frac{x_{i}}{3 z}<r_{i}<\frac{x_{i}}{2 z} \quad \text { for } i=1,2
$$

Then,

$$
\begin{array}{rlr}
6 y_{i} & =\left(3 x_{i}-\sqrt{9 x_{i}^{2}-4-A}\right)(3 z+2)-2\left(3 x_{i}-\sqrt{9 x_{i}^{2}-4-A}\right)+6 r_{i} & \text { for } i=1,2 . \\
& =\left(3 x_{i}-\sqrt{9 x_{i}^{2}-4-A}\right) m n-2\left(3 x_{i}-\sqrt{9 x_{i}^{2}-4-A}\right)+6 r_{i} \quad \text { for } i=1,2 .
\end{array}
$$

So,

$$
\begin{aligned}
6 \mathrm{Tm} & =6 \mathrm{y}_{1}+6 \mathrm{y}_{2}+6 \mathrm{x}_{1}+6 \mathrm{x}_{2} \\
& =\left(3 \mathrm{x}_{1}-\sqrt{9 \mathrm{x}_{1}^{2}-4-\mathrm{A}}+3 \mathrm{x}_{2}-\sqrt{9 \mathrm{x}_{2}^{2}-4-\mathrm{A}}\right) \mathrm{mn}+\mathrm{r}
\end{aligned}
$$

for

$$
r=6 r_{1}+6 r_{2}+2\left(\sqrt{9 x_{1}^{2}-4-A}+\sqrt{9 x_{2}^{2}-4-A}\right)
$$

Since $\mathrm{x}<\mathrm{y}<\mathrm{z}$. So, $\frac{\mathrm{x}}{\mathrm{z}}<1$. Therefore $0<\mathrm{r}_{\mathrm{i}}<\frac{\mathrm{x}_{\mathrm{i}}}{2 \mathrm{z}}<\frac{1}{2}$ for $\mathrm{i}=1$, 2. Also, $\mathrm{x}_{1}<\mathrm{x}_{2}$. Therefore,

$$
\begin{aligned}
|r| & \leq 6\left|r_{1}\right|+6\left|r_{2}\right|+2\left(\sqrt{9 x_{1}^{2}-4-A}+\sqrt{9 x_{2}^{2}-4-A}\right) . \\
& \leq 6\left(\frac{1}{2}\right)+6\left(\frac{1}{2}\right)+2\left(3 x_{1}+3 x_{2}\right) . \\
& =6\left(1+x_{1}+x_{2}\right) . \\
& \leq 6\left(x_{2}+x_{2}\right) \quad \text { being } x_{1}<x_{2} \Rightarrow\left(x_{1}+1\right) \leq x_{2} . \\
& =12 x_{2} .
\end{aligned}
$$

Therefore (8) follows,

$$
\left|\left(3 \mathrm{nx}_{1}-\mathrm{n} \sqrt{9 \mathrm{x}_{1}^{2}-4-\mathrm{A}}+3 \mathrm{nx}_{2}-\mathrm{n} \sqrt{9 \mathrm{x}_{2}^{2}-4-\mathrm{A}}-6 \mathrm{~T}\right) \mathrm{m}\right| \leq 12 \mathrm{x}_{2}
$$

Let,

$$
A^{*}=-n, B^{*}=-n, C^{*}=3 n x_{1}+3 n x_{2}-6 T, M_{i}^{*}=9 x_{i}^{2}-4 \text { for } i=1,2 .
$$


Then $\left[\left(A^{*}\right)^{2} M_{1}{ }^{*}+\left(B^{*}\right)^{2} M_{2}{ }^{*}-\left(C^{*}\right)^{2}\right]^{2}-4\left(A^{*}\right)^{2}\left(B^{*}\right)^{2} M_{1}{ }^{*} M_{2}{ }^{*}$ is divisible by 4. Since $n>0$, so $\left(A \sqrt{M_{1}}+B^{*} \sqrt{M_{2}}\right)<0$. Clearly, neither $\left(9 x_{1}{ }^{2}-4-A\right)$ nor $\left(9 x_{2}{ }^{2}-4-A\right)$ is square. Therefore, by lemma-3 we have $\left(A^{*} \sqrt{M_{1}}+B^{*} \sqrt{M_{2}}+C^{*}\right) \neq 0$. Then by lemma-2, being $4 \leq 4\left|A^{*} B^{*}\right| \sqrt{M^{*} N^{*}+1}$,

$$
\begin{aligned}
\mid A^{*} \sqrt{M_{1}} & +B^{*} \sqrt{M_{2}{ }^{*}}+C^{*} \mid \\
& \geq \frac{4}{\left(2\left|A^{*}\right| \sqrt{M_{1}{ }^{*}}+2\left|B^{*}\right| \sqrt{M_{2}{ }^{*}}+1\right)\left(4\left|A^{*} B^{*}\right| \sqrt{\left.M_{1}{ }^{*} M_{2}{ }^{*}+1\right)}\right.} \\
& \geq \frac{4}{\left(2 n\left(\sqrt{9 x_{1}}+\sqrt{9 x_{2}^{2}}\right)+1\right)\left(4 n^{2} \sqrt{81 x_{1}^{2} x_{2}^{2}}+1\right)} \\
& =\frac{4}{\left(6 n x_{1}+6 n x_{2}+1\right)\left(36 n^{2} x_{1} x_{2}+1\right)}
\end{aligned}
$$

Then (9) becomes with the help of (10),

$$
\mathrm{m} \leq \frac{12 \mathrm{x}_{2}}{4}\left(6 \mathrm{nx}_{1}+6 \mathrm{nx}_{2}+1\right)\left(36 \mathrm{n}^{2} \mathrm{x}_{1} \mathrm{x}_{2}+1\right)
$$

If $\mathrm{x}_{2} \leq 10^{2} \mathrm{n}$ then with $\mathrm{x}_{1}<\mathrm{x}_{2}$,

$$
\begin{aligned}
& \mathrm{m} \leq 3 \mathrm{x}^{2}\left(12 \mathrm{nx}_{2}\right)\left(36 \mathrm{n}^{2} \mathrm{x}_{2}^{2}\right)=1296 \mathrm{x}_{2}{ }^{4} \mathrm{n}^{3}<10^{4}\left(10^{2} \mathrm{n}\right)^{4} \mathrm{n}^{3} \\
& \Rightarrow \mathrm{m}<10^{15} \mathrm{n}^{7} .
\end{aligned}
$$

Therefore, for $\mathrm{z}>5$, then as in theorem $-3.1[\mathrm{C} 1]$,

$$
\mathrm{m}<(\mathrm{mn})^{13 / 14}
$$

Next, if $\mathrm{x}_{2}>10^{2} \mathrm{n}$ then by $\mathrm{x}_{1}<\mathrm{x}_{2}<\mathrm{y}_{2}<\mathrm{z}$ and lemma -4 ,

$$
\begin{aligned}
& \mathrm{m} \leq \mathrm{Tm}=\mathrm{x}_{1}+\mathrm{x}_{2}+\mathrm{y}_{1}+\mathrm{y}_{2} \\
& \leq \mathrm{y}_{1}+3 \mathrm{y}_{2}-3 \\
&\left.<\frac{\mathrm{z}}{3 \mathrm{x}_{1}-1}+\frac{3 \mathrm{z}}{3 \mathrm{x}_{2}-1}-3\right) \\
&=\frac{3 \mathrm{z}+2}{9 \mathrm{x}_{1}-3}+\frac{3 \mathrm{z}}{3 \mathrm{x}_{2}-1}-\frac{2}{9 \mathrm{x}_{1}-3}-3 \\
& \leq \frac{3 \mathrm{z}+2}{9 \mathrm{x}_{1}-3}+\frac{3 \mathrm{z}+2}{3 \mathrm{x}_{2}} \\
&<\frac{\mathrm{mn}}{9 \mathrm{x}_{1}-3}+\frac{\mathrm{mn}}{10^{2} \mathrm{n}} \\
& \Rightarrow \frac{\mathrm{n}}{9 \mathrm{x}_{1}-3}>1-\frac{\mathrm{n}}{10^{2} \mathrm{n}}=1-\frac{1}{10^{2}} \\
& \Rightarrow 9 \mathrm{x}_{1}-3<\frac{\mathrm{n}}{1-10^{-2}} \\
& \Rightarrow \mathrm{x}_{1}<\frac{\mathrm{n}}{9\left(1-10^{-2}\right)}+\frac{1}{3}<\frac{\mathrm{n}}{8}+\frac{1}{3}<\frac{\mathrm{n}}{2}
\end{aligned}
$$


If $x_{2} \leq 2 n^{2} x_{1}$ then we get $x_{2}<n^{3}$ being $x_{1}<\frac{n}{2}$. And $x_{2}>10^{2} n$ follows that $n>10$. Then by (11) we have,

$$
\begin{aligned}
\mathrm{m} & \leq 3 \mathrm{x}_{2}\left(6 \mathrm{nx}_{1}+6 \mathrm{nx}_{2}+1\right)\left(36 \mathrm{n}^{2} \mathrm{x}_{1} \mathrm{x}_{2}+1\right) \\
& \leq 3\left(2 \mathrm{x}_{1} \mathrm{n}_{2}\right)\left(6 \mathrm{nx}_{1}+12 \mathrm{n}^{2} \mathrm{x}_{1}+1\right)\left(72 \mathrm{n}^{4} \mathrm{x}_{1}{ }^{2}+1\right) \\
& =5184 \mathrm{n}^{9} \mathrm{x}_{1}{ }^{4}\left(1+\frac{1}{2 \mathrm{n}^{2}}+\frac{1}{12 \mathrm{x}_{1} \mathrm{n}^{3}}\right)\left(1+\frac{1}{72 \mathrm{n}^{4} \mathrm{x}_{1}{ }^{2}}\right) \\
& <5184 \mathrm{n}^{9}\left(\frac{\mathrm{n}}{9\left(1-10^{-2}\right)}+\frac{1}{3}\right)^{4}\left(1+\frac{1}{2 \mathrm{n}^{2}}+\frac{1}{12 \mathrm{x}_{1} \mathrm{n}^{3}}\right)\left(1+\frac{1}{72 \mathrm{n}^{4} \mathrm{x}_{1}^{2}}\right) \\
& <\frac{5184}{6302} \mathrm{n}^{13}\left(1+\frac{9\left(1-10^{-2}\right)}{3 \mathrm{n}}\right)^{4}\left(1+\frac{1}{2 \mathrm{n}^{2}}+\frac{1}{12 \mathrm{x}_{1} \mathrm{n}^{3}}\right)\left(1+\frac{1}{72 \mathrm{n}^{4} \mathrm{x}_{1}^{2}}\right) \\
& <\mathrm{n}^{13} \\
\Rightarrow & \mathrm{m}^{1 / 14}<\mathrm{n}^{13 / 14} \\
\Rightarrow & \mathrm{m} \cdot \mathrm{m}^{-13 / 14}<\mathrm{n}^{13 / 14} \\
\Rightarrow & \mathrm{m}<(\mathrm{mn})^{13 / 14}
\end{aligned}
$$

Next, assume that $\mathrm{x}_{2}>2 \mathrm{x}_{1} \mathrm{n}^{2}$. By $\left(3 \mathrm{x}_{1}-1\right)<\sqrt{9 \mathrm{x}_{1}{ }^{2}-4-\mathrm{A}}<3 \mathrm{x}_{1}$, we have $\left(9 \mathrm{x}_{1}{ }^{2}-4-\mathrm{A}\right)$ is not a square for $A \leq\left(4 x^{2}-4\right)$. Then $\left[3 n x_{1}-\sqrt{9 x_{1}^{2}-4-A}-6 T\right]$ is irrational and is non-zero. Here,

$$
\begin{aligned}
& n^{2}\left(9 x_{1}^{2}-4-A\right)-\left(3 n x_{1}-6 T\right)^{2} \\
& =9 x_{1}^{2} n^{2}-4 n^{2}-A n^{2}-9 x_{1}{ }^{2} n^{2}-36 T^{2}+36 x_{1} n T \\
& =36 x_{1} n T-4 n^{2}-A n^{2}-36 T^{2}
\end{aligned}
$$

Then by lemma -1, we have,

$$
\left|3 \mathrm{nx}_{1}-\mathrm{n} \sqrt{9 \mathrm{x}_{1}{ }^{2}-4-\mathrm{A}-6 \mathrm{~T}} \geq \frac{2}{6 \mathrm{nx}_{1}+1}\right|
$$

By $x_{2}>2 x_{1} n^{2}$ we have,

$$
\begin{aligned}
\left|3 n x_{2}-n \sqrt{9 x_{2}^{2}-4-A}\right| & =\frac{n+A n}{3 x_{2}+\sqrt{9 x_{2}^{2}-4-A}} \\
& \leq \frac{n+A n}{12 x_{1} n^{2}+3} \\
& \leq \frac{x^{2}}{x_{1} n+1} \quad\left[\text { Being } A \leq 4\left(x^{2}-1\right)\right]
\end{aligned}
$$

Therefore,

$$
\begin{aligned}
& \left|3 n x_{1}-n \sqrt{9 x_{1}^{2}-4-A}+3 \mathrm{nx}_{2}-\mathrm{n} \sqrt{9 \mathrm{x}_{2}^{2}-4-\mathrm{A}}-6 \mathrm{~T}\right| \mathrm{m} \\
& \geq \frac{2 \mathrm{~m}}{6 \mathrm{nx}_{1}+1}-\frac{\mathrm{m}+\mathrm{Am}}{12 \mathrm{n}^{2} \mathrm{x}_{1}+3} \\
& \geq \frac{2 \mathrm{~m}}{6 \mathrm{nx}_{1}+1}-\frac{\mathrm{x}^{2}}{\mathrm{nx}_{1}+1}
\end{aligned}
$$




$$
\begin{aligned}
& \geq \frac{2 \mathrm{~m}}{6 \mathrm{nx}_{1}+1}-\frac{\mathrm{m}}{6 \mathrm{nx}_{1}+1} \\
& =\frac{\mathrm{m}}{6 \mathrm{nx}_{1}+1}
\end{aligned}
$$

Therefore, by (9) and (13) we have,

$$
\begin{aligned}
& \frac{\mathrm{m}}{6 \mathrm{nx}_{1}+1} \leq 12 \mathrm{x}_{2} \\
\Rightarrow & \mathrm{m} \leq 12 \mathrm{x}_{2}\left(6 \mathrm{nx}_{1}+1\right) \leq 12 \mathrm{x}_{2}\left(7 \mathrm{nx}_{1}\right)=84 \mathrm{x}_{1} \mathrm{x}_{2} \mathrm{n}
\end{aligned}
$$

By lemma $-3.1[\mathrm{C} 1]$,

$$
\mathrm{x}^{2} \leq \frac{\mathrm{z}}{5}<\frac{3 \mathrm{z}}{15}<\frac{3 \mathrm{z}+2}{14}=\frac{\mathrm{mn}}{14}
$$

Since $\mathrm{x}_{1}<\frac{\mathrm{n}}{8}+\frac{1}{3}<\frac{\mathrm{n}}{2}$. So, by (13) and (14) we have [C1],

$$
\begin{aligned}
& \mathrm{m} \leq 84 \mathrm{x}_{1} \mathrm{x}_{2} \mathrm{n}<42 \mathrm{x}_{2} \mathrm{n}^{2}<3 \sqrt{14} \mathrm{~m}^{1 / 2} \mathrm{n}^{5 / 2}<14 \mathrm{~m}^{1 / 2} \mathrm{n}^{5 / 2} \\
& \Rightarrow \mathrm{m}^{1 / 3} \leq 14^{1 / 3} \mathrm{~m}^{1 / 6} \mathrm{n}^{5 / 6} \\
& \Rightarrow \mathrm{m} \leq 14^{1 / 3} \mathrm{~m}^{5 / 6} \mathrm{n}^{5 / 6}<(\mathrm{mn})^{13 / 14} . \\
\text { i:e: } & \operatorname{gcd}\left(\mathrm{x}_{1}+\mathrm{x}_{2}+\mathrm{y}_{1}+\mathrm{y}_{2}, 3 \mathrm{z}+2\right)<(3 \mathrm{z}+2)^{13 / 14} .
\end{aligned}
$$

By lemma $-3.2[C 1]$, we have $\mathrm{y}_{1}+\mathrm{x}_{1}>\mathrm{y}_{2}+\mathrm{x}_{2}$. Similarly we have,

$$
\operatorname{gcd}\left(\left(\mathrm{y}_{1}+\mathrm{x}_{1}\right)-\left(\mathrm{y}_{2}+\mathrm{x}_{2}\right), 3 \mathrm{z}+2\right)<(3 \mathrm{z}+2)^{13 / 14}
$$

Since,

$$
\begin{aligned}
& \left(\mathrm{x}_{1}+\mathrm{x}_{2}+\mathrm{y}_{1}+\mathrm{y}_{2}\right)\left[\left(\mathrm{y}_{1}+\mathrm{x}_{1}\right)-\left(\mathrm{y}_{2}+\mathrm{x}_{2}\right)\right] \\
& =\left(\mathrm{y}_{1}+\mathrm{x}_{1}\right)^{2}-\left(\mathrm{y}_{2}+\mathrm{x}_{2}\right)^{2} \\
& =\mathrm{x}_{1}{ }^{2}+\mathrm{y}_{1}{ }^{2}+2 \mathrm{x}_{1} \mathrm{y}_{1}-\mathrm{x}_{2}{ }^{2}-\mathrm{y}_{2}{ }^{2}-2 \mathrm{x}_{2} \mathrm{y}_{2} \\
& =\mathrm{z}^{2}+3 \mathrm{x}_{1} \mathrm{y}_{1} \mathrm{z}+2 \mathrm{x}_{1} \mathrm{y}_{1}-\mathrm{z}^{2}-3 \mathrm{x}_{2} \mathrm{y}_{2} \mathrm{z}-2 \mathrm{x}_{2} \mathrm{y}_{2} \\
& =(3 \mathrm{z}+2)\left(\mathrm{x}_{1} \mathrm{y}_{1}-\mathrm{x}_{2} \mathrm{y}_{2}\right) .
\end{aligned}
$$

This follows that $(3 \mathrm{z}+2)$ divides $\left[\left(\mathrm{y}_{1}+\mathrm{x}_{1}\right)-\left(\mathrm{y}_{2}+\mathrm{x}_{2}\right)\right]$. So,

$$
(3 \mathrm{z}+2)^{1 / 14}<\operatorname{gcd}\left(\left(\mathrm{y}_{1}+\mathrm{x}_{1}\right)-\left(\mathrm{y}_{2}+\mathrm{x}_{2}\right), 3 \mathrm{z}+2\right) .
$$

From (16) and (17), the proof of the theorem is completed.

Theorem - 4.2(a): If $3 \mathrm{z}+2$ is of the form $\mathrm{kp}^{l}$ where $\mathrm{p}^{l}$ is a prime power and $\mathrm{k}$ is a positive integer with $\mathrm{k}<(3 \mathrm{z}+2)^{1 / 14}$ then $\mathrm{z}$ is unique.

Proof: Suppose $3 \mathrm{z}+2=\mathrm{kp}^{l}$ where $\mathrm{p}^{l}$ is a prime power and $\mathrm{k}$ is a positive integer with $\mathrm{k}<(3 \mathrm{z}+2)^{1 / 14}$. If $\mathrm{z}$ is not unique then there is two Markoff triples $\left(\mathrm{x}_{1}, \mathrm{y}_{1}, \mathrm{z}\right)$ and $\left(\mathrm{x}_{2}, \mathrm{y}_{2}, \mathrm{z}\right)$ with $\mathrm{x}_{1}<\mathrm{y}_{1}<\mathrm{z}$ and $\mathrm{x}_{2}<\mathrm{y}_{2}$ $<\mathrm{z}$. Recall that $\mathrm{z}>10^{140}$, we have

$$
\mathrm{p}^{l}>(3 \mathrm{z}+2)^{13 / 14}>10^{130}
$$


By Zhang [[Z2], Theorem 1] we have 4 divides $(3 z+2)$. So, $p>2$. Thus, $p$ does not divides $z$. Since

$$
\left(y_{i}+x_{i}\right)^{2}+z^{2}-A=(3 z+2) x_{i} y_{i}=k^{l} x_{i} y_{i} \quad \text { for } \mathrm{i}=1,2 .
$$

Then

$$
\begin{aligned}
& \left(\mathrm{y}_{1}+\mathrm{x}_{1}\right)^{2}-\left(\mathrm{y}_{2}+\mathrm{x}_{2}\right)^{2}=\mathrm{kp}^{l}\left(\mathrm{x}_{1} \mathrm{y}_{1}-\mathrm{x}_{2} \mathrm{y}_{2}\right) \\
\Rightarrow & \left(\mathrm{x}_{1}+\mathrm{x}_{2}+\mathrm{y}_{1}+\mathrm{y}_{2}\right)\left[\left(\mathrm{y}_{1}+\mathrm{x}_{1}\right)-\left(\mathrm{y}_{2}+\mathrm{x}_{2}\right)\right]=\mathrm{kp}^{l}\left(\mathrm{x}_{1} \mathrm{y}_{1}-\mathrm{x}_{2} \mathrm{y}_{2}\right) .
\end{aligned}
$$

This imply either, $\mathrm{p}^{l}$ divides $\left(\mathrm{y}_{1}+\mathrm{x}_{1}\right)\left(\mathrm{y}_{2}+\mathrm{x}_{2}\right)$. Therefore, by theorem -3.1 (See [C1])

$$
\text { or } \quad \operatorname{gcd}\left(\left(\mathrm{y}_{1}+\mathrm{x}_{1}\right)-\left(\mathrm{y}_{2}+\mathrm{x}_{2}\right), 3 \mathrm{z}+2\right) \geq \mathrm{p}^{l}>(3 \mathrm{z}+2)^{13 / 14} .
$$

This leads a contradiction to theorem -6 . So, $\mathrm{z}$ should be unique.

In similar way to theorem 7(a) we can prove the following theorem.

Theorem -7(b): If $3 \mathrm{z}-2$ is of the form $\mathrm{kp}^{l}$ where $\mathrm{p}^{l}$ is a prime power and $\mathrm{k}$ is a positive integer with $\mathrm{k}<(3 \mathrm{z}-2)^{1 / 14}$ then $\mathrm{z}$ is unique.

\section{References}

1. Chen, F.J. and Y.G. Chen, "On the Frobenius Conjecture for Markoff Numbers", J. Number Theory, Vol.133(2013), pp. 2363-2373.

2. Zhang, Y., "An Elementary Proof of Uniqueness of Markoff Numbers Which Are Prime Powers", (2007). arXiv: math/0606283v2.

3. Barager, A., "On the Unicity Conjecture for Markoff Numbers", Canad. Math. Bull., vol.39(1996), pp. 3-9.

4. Button, J.O., "Markoff Numbers, Principal Ideals and Continued Fraction Expansions", J. Number Theory, Vol.87(2001), pp. 27-44.

5. Srinivasan, A., "Markoff Numbers and Ambiguous Classes", J. Theor. Numbers Bordeaux, Vol.21(2009), No. 3, pp. 755-768 (English version: MR2605546 (2011e:11118).

6. Mcginn, D.J., “Generalized Markoff Equations, Euclid Trees, and Chebyshev Polynomials”, $\mathrm{PhD}$ Thesis, University of Nevada, Las Vegas, 2015.

7. Zhang, Y., "Congruence and Uniqueness of Certain Markoff Numbers", Preprint, arXiv: math.NT/0612620. 